Postprint

Published in: Polymer testing 91 (2020) 106799

DOI: https://doi.org/10.1016/j.polymertesting.2020.106799

\title{
Influencing parameters on measurement accuracy in dynamic mechanical analysis of thermoplastic polymers and their composites
}

Joanna Schalnat ${ }^{1}$, David Garoz Gómez ${ }^{1,2}$, Lode Daelemans ${ }^{1}$, Ives De Baere ${ }^{1}$, Karen De Clerck ${ }^{1}$ and Wim Van Paepegem ${ }^{1 *}$

${ }^{1}$ Department of Materials, Textiles and Chemical Engineering (MaTCh), Ghent University, TechnologieparkZwijnaarde 46/70, B-9052 Zwijnaarde, Belgium

${ }^{2}$ IMDEA Materials, c/Erik Kandel 2, Parque Científico y Tecnológico - Tecnogetafe, 28906 Getafe, Madrid, Spain.

*Wim.VanPaepegem@UGent.be,Tel.: +32-(0)9-331.04.32, Fax: +32-(0)9-264.58.33

\begin{abstract}
Long-term predictions of material properties such as stiffness and creep resistance are important in many engineering applications and require high reliability and accuracy. This is especially true for polymer materials and their composites as their viscoelastic nature results in timedependent material behaviour and any measurement uncertainties or errors amplify in longterm predictions. To measure this behaviour at smallest loadings, Dynamic Mechanical Analysis (DMA) is frequently declared as an ideal method. However, the measurement accuracy and repeatability of this method is strongly influenced by (i) the testing fixture and corresponding loading mode, (ii) the sample preparation and (iii) the plotting scale to interpret the test results. In this study, relevant experimental parameters were found for DMA and a proper procedure was designed, which was then applied to measure the viscoelastic behaviour of a highly temperature and creep resistant thermoplastic polymer (polyethersulfone) and of a highly graphite filled polypropylene composite. In combination with finite element simulations and in-situ strain measurements by digital image correlation (DIC), the main influences on measurement accuracy of three-point-bending DMA were identified and subsequently used to determine measurement guidelines. Using these guidelines, DMA measurements allow
\end{abstract}


quantitative determination of the viscoelastic response for rigid polymer and composite materials.

Keywords: DMA, accuracy, repeatability, 3-point-bending, PESU, Technoform-PP

\section{Introduction}

Dynamic Mechanical Analysis (DMA) is a technique to characterise the viscoelastic behaviour of a material as function of time, temperature, frequency, or stress. In a specific fixture, a specimen is clamped and loaded using very small, sinusoidal waveforms. The technique is predominantly used to study the thermal transition of polymer materials as a function of temperature, for instance to derive the glass transition temperature $\mathrm{T}_{\mathrm{g}}$ [1]. However, it is also possible to use DMA to determine the elastic and viscous response of the material, respectively storage and loss modulus, based on linear viscoelastic theory [2].

As such, the technique is directly capable of determining the linear viscoelastic material response in function of temperature. The data can be used for long-term predictions of material behaviour using for example the principle of Time-Temperature-Superposition (TTS) [3] or as input in numerical simulations, predicting the creep performance of structural components. While older journal articles stress the purely qualitative value of DMA results [4], more recent ones allow for quantitative usage [5], [6], [7], [8]. Especially for long-term predictions, the data is usually plotted on a logarithmic scale, displaying several orders of magnitude in for example modulus values. This logarithmic representation of the data conceals the measurement accuracy at a fixed point in time or temperature [5], [9]. Moreover, the amount of measurement repetitions with DMA is not always mentioned or can be very low [10], [11]. If no precautions are taken (arbitrary settings), the scatter on the acquired data can be very large, in some cases up to $50 \%$. Hence, any long-term predictions based on data with this amount of scatter also results in an unreliable prediction.

In this study, the accuracy of DMA results to obtain quantitative data was analysed and evaluated. Measurement parameters were defined based on a study of published literature (Section 2) and their effect on the results was determined (Section 4). In addition, the experiments were accompanied by finite element analysis and optical strain measurements through digital image correlation to allow a better understanding of the displacements induced by DMA test equipment. This was first done for a PESU polymer material that had high 
temperature resistance (no thermal transitions within the temperature range studied) and could be obtained in a high quality grade ensuring material homogeneity, absence of micro-voids and defects and repeatability. Thereafter, the principles were applied to determine the response of a polymer composite based on polypropylene used in engineering applications.

\section{Overview of possible parameters influencing DMA measurement accuracy}

In the following paragraphs, the testing parameters for DMA in 3-point-bending loading mode are discussed, focussing on the following four aspects: specimen (size, dimensional accuracy), fixture (pre-stress), machine settings (heating rate, amplitude, frequency), and measurement repetition (amount of tests, samples and cycles). Although other loading fixtures such as tensile, compression or cantilever bending exist, these clamp types are typically not suited to determine the viscoelastic response of rigid polymer bulk materials due to the material stiffness and the very limited load range of most DMA equipment.

\subsection{Sample dimensions and dimensional accuracy}

The required sample dimensions differ by instrument, fixture type and material stiffness, but recommendations can be found in the device's manual [1], [2], [12] and testing standards such as ASTM D790 [13] and others [14], [15], [16]. Different manufacturers offer 3-point-bending fixtures for which the samples require similar dimensions. The specimen length is defined by the gauge length of the fixture with addition of a certain overhang of the sample against slippage [14]. The specimen thickness should be chosen such that the resulting bending stiffness is less than a third of that of the fixture [1]. The span length to thickness ratio (ST-ratio) should be at least 10 for pure polymers [15]. Some authors advise large aspect ratios especially for anisotropic [10] or glassy material [17]. Others stress that strict limitations of sample dimensions are required for repeatable measurements [8], [17]. The specimen's width is typically only limited by material or the width of the fixture [2], [18] or considered as of negligible importance [19]. Some standards mention typical specimen dimensions with a width of $12 \mathrm{~mm} \mathrm{[15]} \mathrm{or} 13 \mathrm{~mm} \mathrm{[14]} \mathrm{without} \mathrm{stating} \mathrm{the} \mathrm{reason} \mathrm{for} \mathrm{this} \mathrm{value.}$

The accuracy of the dimensions is described as uniform [6], rectangular [14], accurate [1] and parallel [2], [12], but the exact details remain vague at times. Only very few standards specify that the thickness or width variation should be less than $\pm 2 \%$ [15] respectively $\pm 3 \%$ [16] over the full length measured at five points along its length. Overall, the advised sample size and 
accuracy varies per manufacturer and fixture, but limiting rules and explanations for the boundaries are rarely provided.

\subsection{Fixture}

For samples with a high bending stiffness, cantilever or 3-point-bending fixtures are typically used since the flexural loading allows for higher deformation (e.g. strain level up to $1 \%$ ) at a load within the usually low limits of the instrument's load cell [2]. Since the deformation in bending fixtures is not influenced by clamping effects, this option is to be preferred [4]. Nevertheless, the clamping or bolting of the fixture to the DMA machine itself, could introduce variability in the measurement.

In bending fixtures, a pre-stress is required to prevent the sample from shifting in the fixture. The amount of pre-stress must be well chosen [4], [7], [10], [20]. If the pre-stress is too low, the sample is insufficiently clamped. Whereas if it is too high, the dynamic result will be influenced [10]. Temperature effects can cause a change in properties, which can span several orders of magnitude. If the temperature influence is of interest, the selection of a good pre-stress value becomes even more cumbersome. A pre-stress amplitude valid for a glassy material state might become invalid by an increase of temperature causing creep in the rubbery state of the material. Some instruments deal with this problem using an automatic adjusting static strain [4], [20]. However, this has the drawback that the loading conditions change during the measurement.

\subsection{Settings}

For the mechanical response, it is important to have a homogeneous temperature over the complete sample. Consequently, for non-isothermal experiments, the heating rate can severely affect the results, as a temperature-lag can exist between the core of the specimen and the nearby thermocouple. To ensure a constant temperature, it is either recommended to keep the heating rate lower than $5 \mathrm{~K} / \mathrm{min}$ [1] or to insert a isothermal waiting time (dwell time) of several minutes [1], [7] before the actual test. Self-heating of thicker samples can be avoided by not fully exhausting the available frequency range [5].

All commonly available DMA instruments have a large amplitude range of at least $1000 \mu \mathrm{m}$, but recommended amplitudes are for all kind of fixtures below $50 \mu \mathrm{m}$ [18], [21], [22]. 
Furthermore, for 3-point-bending fixture of TA Instruments the advised amplitudes are narrowed down to $25-40 \mu \mathrm{m}[2]$.

The smaller the amplitude, the more crucial the specimen's geometrical accuracy becomes to ensure correct load transfer from the fixture to the specimen [17]. This is confirmed in the studies of Lee-Sullivan and Shao [4], [23] which conclude that higher amplitude resulted in lower standard deviations of the results. Limitations on the amplitude are dynamic effects, the instrument's upper force limit [2] and higher strains which can fall outside the linear viscoelastic region of the specimen. Also, the occurring strain rate by amplitude and frequency can influence the mechanical response as Margossian [7] showed by comparing the differences in results between $10-500 \mu \mathrm{m} / \mathrm{min}$, showing a stiffer response at higher strain rates.

\subsection{Repetitions}

Most studies do not mention the amount of repetitions per experiment [5], [8], [9], [10], [11], [24]. Some standards propose two specimens per experiment [6], [15]. While some authors re-use one sample for several tests [8], [11], others advise to always take a new sample, even if the test has been performed purely in the viscoelastic region of the material [14]. Also a combination is possible where a sample is taken for each new experiment, but the test is repeated several times in one run, since the first cycle might diverge from the average [4].

\section{Materials and methods}

\subsection{Materials}

To limit the influences on the experiments in the parametric study, a highly creep resistant polymer with low sensitivity to moisture was chosen, i.e. polyether sulfone (PESU, Veradel A301, Solvay). PESU is an amorphous thermoplastic with a glass transition temperature higher than $200^{\circ} \mathrm{C}$ and a Poisson's ratio of 0.41 [25], [26].

The results of this study were verified by using a polypropylene based tubular injected composite with a filler content of $75 \mathrm{wt} . \% / 50$ vol.\% graphite flakes, i.e. Technoform PP from the company Technoform [27]. The material has its melting point at $165{ }^{\circ} \mathrm{C}$ and a Poisson's ratio of 0.24 . For testing purposes, the $24.5 \mathrm{~mm}$ diameter tube was cut in sections. The outer part of the tube section was always loaded in compression in 3-point-bending. The change in moment of inertia for the specimens cut from tubular material compared to perfect rectangular 
beams was negligible $(<0.32 \%)$. Due to its application in heat exchangers, the thermomechanical properties and the viscoelastic properties of this material in particular are very relevant.

\subsection{Specimen preparation}

All samples were $60 \mathrm{~mm}$ long. The thickness was given by the production process, for PESU $2.09 \pm 0.01 \mathrm{~mm}$ and for Technoform-PP $1.57 \pm 0.01 \mathrm{~mm}$. This results into a span to thickness ratio of 24 (50 mm 3-point-bending fixture) and 32 respectively. The width resulted from the manual cutting preparation process with a maximum variation over the length of one sample of $1.5 \%$, which is a divergence of less than $\pm 0.05 \mathrm{~mm}$. The cut edges were polished with grinding paper down to $5-\mu \mathrm{m}$ grain size to ensure the accurate geometry; the angles were measured and any variability was limited to $90.0 \pm 0.5^{\circ}$, verified through optical microscopic measurements. Due to the curvature of the tube sector, the angle of the Technoform-PP samples was not as precise $\left(90 \pm 3^{\circ}\right)$ as for the flat PESU, samples and the width varied more amongst different samples (Figure 1). However, the precision of the width within one individual sample was the same as for the flat samples.

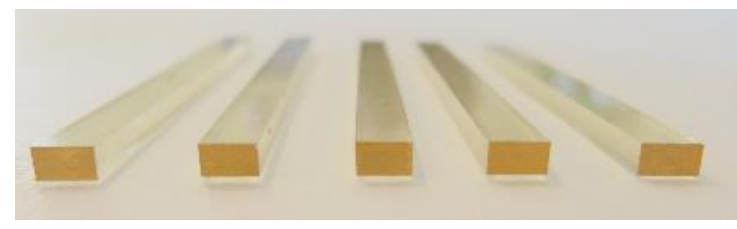

(a)

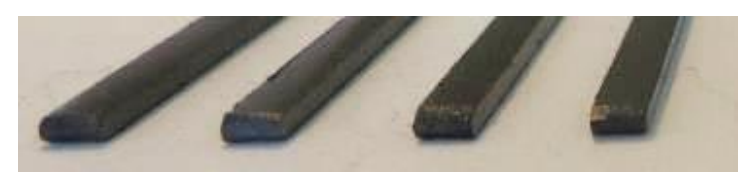

(b)

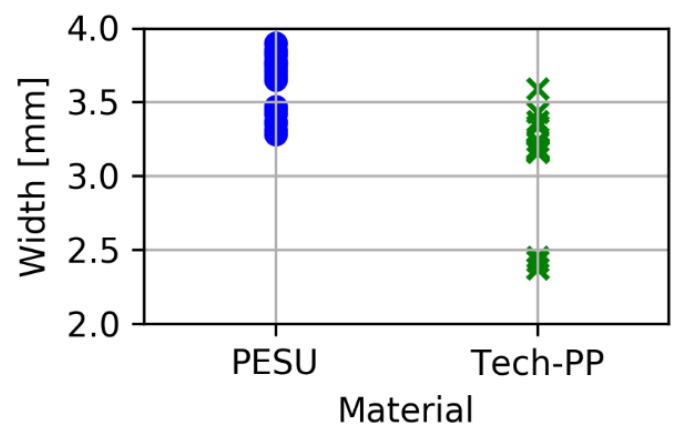

(c)

Figure 1: Accurately cut DTMA-samples: (a) PESU, (b) Technoform-PP graphite-filled PP samples and (c) the variation of the width amongst different samples (each sample on its own has a variation of less than $1.5 \%$ )

\subsection{Testing procedure}

Dynamic Mechanical Analysis was performed on a TA Instruments DMA Q800 [22] set-up using a $50 \mathrm{~mm}$ 3-point-bending fixture with support rolls with a diameter of $3.15 \mathrm{~mm}$ and $15 \mathrm{~mm}$ span clamp with $0.4 \mathrm{~mm}$ rolls. Tests were conducted at $35^{\circ} \mathrm{C}$ to ensure the machine reached a stable temperature without the necessity for active liquid nitrogen cooling. 
In DMA, several tests with parameter sweeps can be performed: amplitude, frequency, and temperature sweep. Literature's recommendation for settings provides machine-specific settings for every sweep. To create material-specific testing parameters, results from one parameter sweep provide guiding values for others. A testing procedure was established to define the relevant parameters and limitations according to the requirements of the tested material (Figure 2). First, an amplitude sweep is performed to determine the stable linearviscoelastic (LVE) region of the material and to choose a suitable amplitude to perform the successive frequency sweep. The purpose of the frequency sweep is either to determine the viscoelastic properties at a fixed temperature or to be the basis for the TTS [3]. For the latter, a subsequent temperature sweep is performed to detect thermal transitions of the material.

The parameters are influencing each other partially; therefore, sweeps can be looped to iterate the optimal testing conditions.

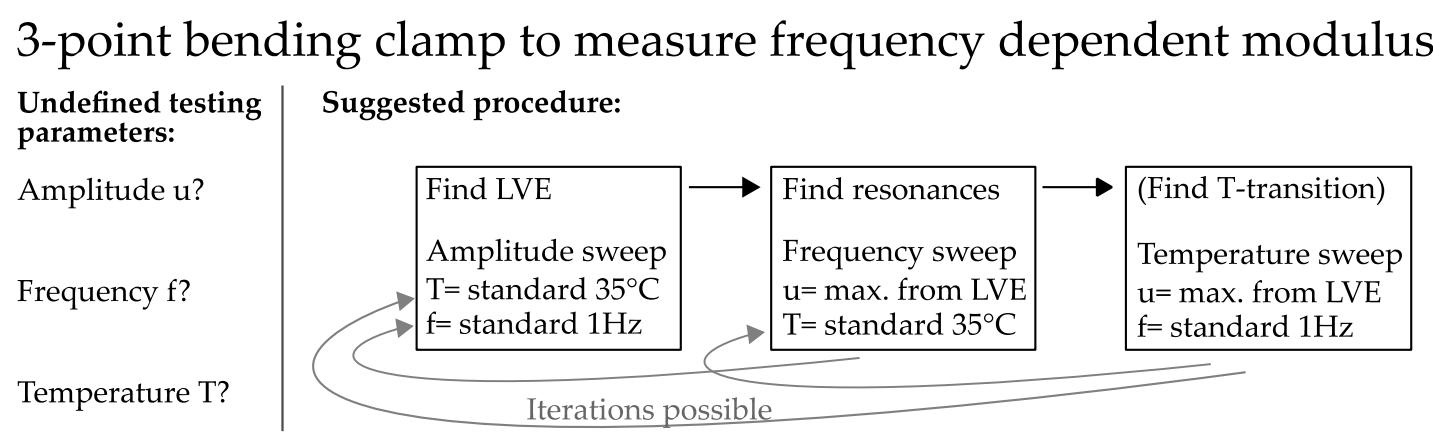

Figure 2: Developed testing procedure based on PESU samples

\subsection{Calculations}

The storage modulus (E') and loss modulus (E') were chosen as representative results from the DMA test. As in every DMA device, the storage modulus (E') is calculated as in equation (1) from stress $(\sigma)$ and strain ( $\varepsilon$. As equation (1) [28] shows, the modulus is based on the force (F) loading the sample, the displacement (u), the Poisson's ratio (v), the energy dissipation $\tan \delta$ as well as the dimensions of the sample: gauge length (L), width (w) and thickness (t).

$$
E^{\prime}=\frac{\sigma}{\varepsilon} \cos (\tan \delta)=\frac{F L^{3}\left[1+\frac{12}{5}(1+v)\left(\frac{t}{L}\right)^{2}\right]}{4 u w t^{3}} \cos (\tan \delta)
$$

To express the systematic error of the measurement, the relative error is used. This error is the same for all tests conducted in the same system. 
Different results were compared in terms of their deviation by using the standard deviation. To be able to compare the results of different materials with different moduli, the standard deviation was divided by the average to provide a deviation from the average in percent, also called scatter in this paper.

\subsection{Independent strain measurements through digital image correlation}

The strain was also measured externally to analyse its distribution over the sample. Digital Image Correlation (DIC), a visual, contactless, and full-field 3D deformation measurement technique, was utilised to visualise strain distribution over the sample and to verify the movement of the drive shaft (Figure 3). Two cameras trace the sample, which has an applied optical pattern. In the post-processing, the images are compared over time and the differences are correlated to strain. For this purpose, a fine speckle pattern was applied to the sample and to the loading nose, which allowed tracking of the deformation and movements. The results were analysed using Vic-3D [29].

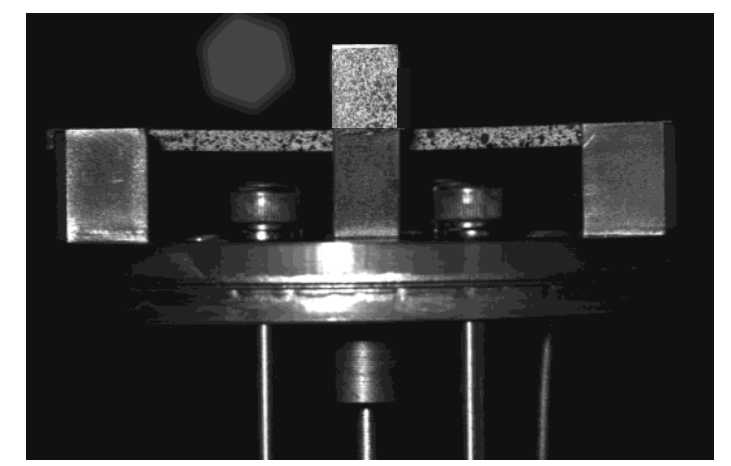

Figure 3: 3-point-bending fixture with DIC speckle pattern on the loading nose and the specimen

\subsection{Numerical framework}

A 3D finite element model (FEM) was constructed using the commercial software ABAQUS [30]. The model replicates the dynamic 3-point-bending-test using realistic material models, dimensions and boundary conditions. The viscoelastic behaviour in each material point is defined by the instantaneous elastic properties and the Prony series in the time domain. The material input parameters have been determined from the experimental campaign at the reference temperature. The geometry of the specimen and the position of the holders correspond to the experimental setup. The dynamic response of the virtual test is processed as the real experimental test: the displacement and reaction force outputs in the time domain are converted into frequency domain with the Fast Fourier Transformation to determine the phase lag. The numerical dynamic response is obtained using a dynamic implicit solver. The convergence 
study has determined the size of the finite elements along the specimen and around the contact areas, see finite element mesh in Figure 4. To avoid issues with the numerical contacts between holders and the specimen, they have been simplified with displacement boundary conditions.

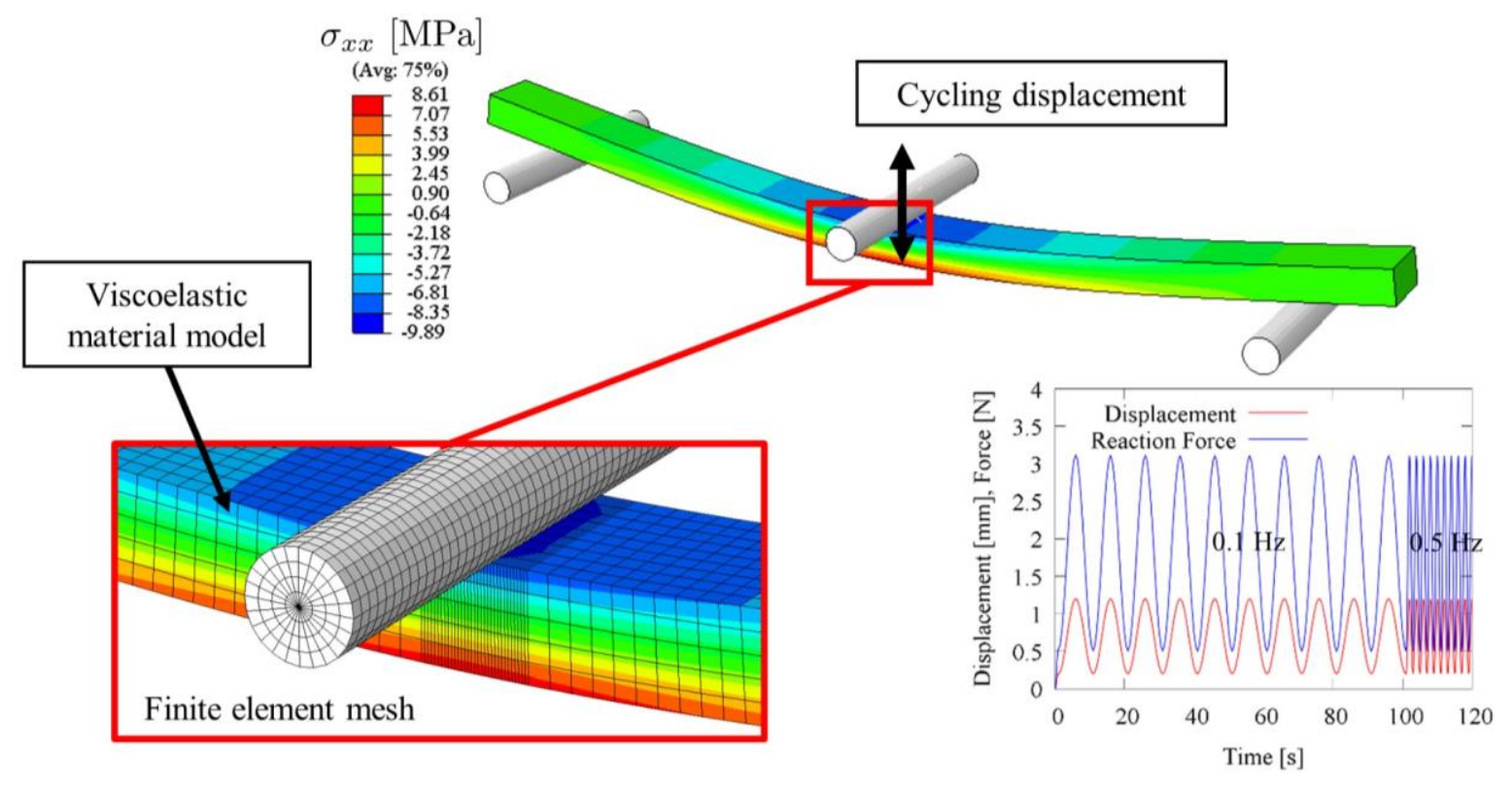

Figure 4. 3D finite element model of the 3-point-bending-test under cycling displacement. Mesh refined in the contact areas with supports and shaft. Dynamic response of the reaction force at different frequencies.

\section{Results and Discussion}

\subsection{Development of generic guidelines based on PESU}

In order to develop a system for more accurate DMA measurements, different parameters were varied on the pure polymer PESU. In the following, the scatter of a result is used only for comparison within one graph. For each graph, the tests were performed with the same settings except the one parameter that was under investigation. Between different graphs, a comparison is not valid, since several parameters can be different.

\subsubsection{Dimensional accuracy of specimens}

Using the specimen dimensions and dimensional accuracy, the highest relative error on the storage modulus was calculated using Equation 1 to be maximal $2.0 \%$ ( $\pm 56 \mathrm{MPa}$ ) for PESU. However, initial DMA experiments on specimens with varying widths on PESU showed a standard deviation of more than $\pm 104 \mathrm{MPa}$. This indicates that other factors than the specimen's precision are indeed influencing the modulus as well. As there is rarely any guideline provided 
in test standards for the specimen width, narrow and wide specimens were compared (Figure 5). The narrow PESU samples were $3.6 \mathrm{~mm}$ wide, the wide ones $7.6 \mathrm{~mm}$. Narrow samples have a slightly lower average $(2902 \pm 63 \mathrm{MPa})$ than wide samples $(2932 \pm 54 \mathrm{MPa})$. The FE simulation confirms the trend that narrow samples have a slightly lower storage modulus (2843 MPa) than wide samples (2894 MPa). The experimental tests have a scatter of $2.2 \%$ (narrow samples) and $1.8 \%$ (wide samples).

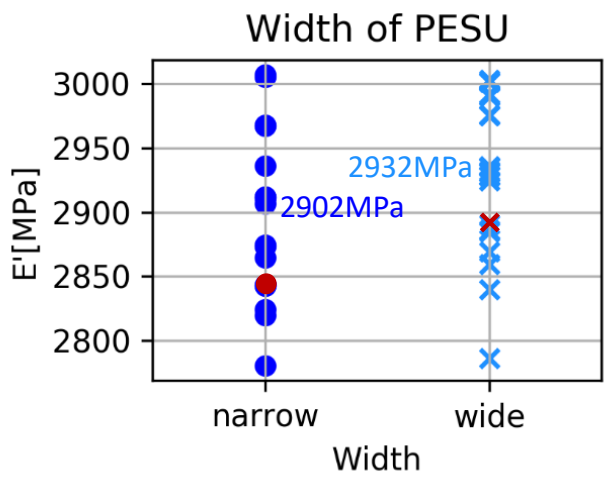

Figure 5: Effect of the sample width on the storage modulus. Each symbol corresponds to a measurement on a single specimen (20 per width), average written next to group. Simulation values for PESU in red.

\subsubsection{Fixture type and clamping conditions}

The specimen length is fixed to $60 \mathrm{~mm}$, but the span length depends on the clamp. As to see in Error! Reference source not found.a, the specific clamp has flexible rolls that can rotate within the clamp. This can cause an offset of up to $50 \pm 1 \mathrm{~mm}$ on the span, which would result in an error of $6 \%$ on the storage modulus. However, for testing, the rolls were manually readjusted to have a very limited shift. Therewith the error could be kept below $0.6 \%$.

The fixture that holds the sample is attached to the DMA machine using four bolts (Figure 6a). Split washers underneath the bolt help to maintain the applied torque. The effect of fixing these bolts to the machine was compared for (i) hand tightened bolts without measuring control and likely, below $0.1 \mathrm{Nm}$; (ii) bolts randomly tightened with a torque wrench, giving each bolt a randomly different torque in the range $0.1-1.7 \mathrm{Nm}$; and, (iii) all bolts tightened with a torque of $1.01 \mathrm{Nm}$. For each setting, the machine compliance was calibrated and resulted in similar values within the machine specific recommended region. The same set of five samples was taken for each of the three settings, allowing recovery time and random order to exclude influences. After the test, the torque wrench fixed bolts were tested and showed the same amount of torque before and after the test. 
The results are given in Figure $6 \mathrm{~b}$. The tests with a torque of $1.01 \mathrm{Nm}$ show an almost constant modulus from the beginning of the initiation phase until the end of the testing phase (here 5 minutes). Randomly tightened bolts in the range 0.1-1.7 Nm showed a higher modulus in the start-up period, but in the testing phase, the modulus was similar to the tests at $1.01 \mathrm{Nm}$ except for one outlier. Hand-tightened bolts however resulted in an overall lower modulus, even though the modulus was constant from the beginning of the test on. The scatter on the average storage modulus was with $2.4 \%$ scatter for bolts tightened with $1.01 \mathrm{Nm}$ slightly lower than for the other methods (3.2\% for random $0.1-1.7 \mathrm{Nm}$ and $3.4 \%$ for hand-tight). For the initialising phase, equally tightened bolts result in a steadier modulus from the beginning of the test on compared to the randomly tightened bolts. For the testing phase, a torque between 0.1 $1.7 \mathrm{Nm}$ should be chosen for a repeatable value of the modulus.

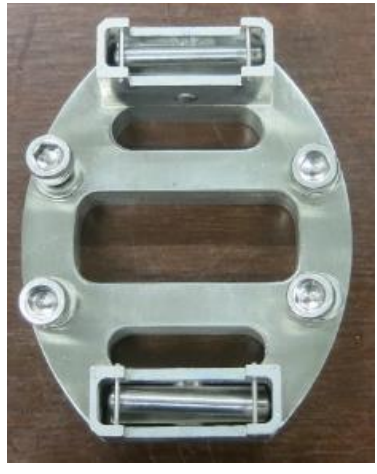

(a)

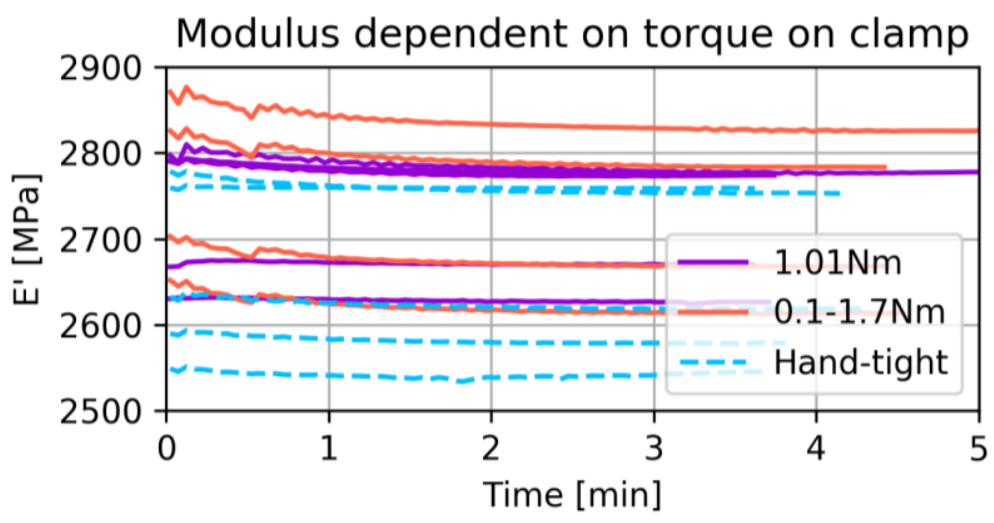

(b)

Figure 6: Influence of the torque values of the fixture on the storage modulus. (a) 50 mm fixture from TA Instruments with movable support beams and four fixture bolts (b) Plot of the effect on PESU samples with bolts tightened by hand without force control, tightened individually randomly in the range of 0.1 $1.7 \mathrm{Nm}$ and uniform torque of $1.01 \mathrm{Nm}$ on all bolts. Five samples per group.

For the ideal holding of the sample, two different settings can be defined in the TA Q800 to hold the sample in place. The pre-force is a fixed value that should be chosen similar to the expected force level during the test to ensure clamping, but prevent breakage. Pre-force is always applied as a first step in the experiment. In the initiation phase, the static force can be replaced by a force track. This setting adjusts the static force dynamically as a percentage to the dynamic force that is applied to test the sample. Especially, when the modulus of the sample changes due to circumstances like temperature, the flexible method helps to stay in the linear viscoelastic region of the sample. The static force should be bigger than the dynamic force to ensure clamping during all times. The standard value for the static force is $125 \%$ of the dynamic force. It is compared in Table 1 with $101 \%$ and $200 \%$ to see if the standard value is suited for a stiff sample in 3-point-bending. The different settings were applied to the same set of five 
samples in random order and with sufficient recovery time. The modulus and scatter values between these tests are overall very similar. There is however a decreasing trend visible in the storage modulus and an increasing trend in the loss modulus for increased values of the static force. Yet high static force need to be avoided to not risk to test outside of the linear viscoelastic region. The standard setting of $125 \%$ compared to the dynamic force is retained for all following tests.

Table 1: Average, standard deviation and scatter of storage and loss modulus dependent on the amount of static force during the dynamic test on narrow PESU-samples

\begin{tabular}{llll}
\hline $\begin{array}{l}\text { Static force (with } \\
\text { respect to dynamic } \\
\text { force) }\end{array}$ & $101 \%$ & $125 \%$ & $200 \%$ \\
\hline $\begin{array}{l}\text { Average E' } \\
\text { STDEV E' }\end{array}$ & 2931 & 2920 & 2911 \\
Scatter E' & 61 & 60 & 57 \\
& $2.1 \%$ & $2.0 \%$ & $2.0 \%$ \\
Average E', & 26.8 & 27.3 & 28.6 \\
STDEV E', & 0.7 & 0.7 & 1.0 \\
Scatter E", & $2.7 \%$ & $2.5 \%$ & $3.4 \%$ \\
\hline
\end{tabular}

The position of the sample in the 3-point-bending fixture should be centred both in length and in width on the rollers, but manual handling can cause an offset of several degrees. In a static test, as in Figure 7a, even an exaggerated mismatch of $10^{\circ}$ between specimen and fixture line does neither numerically nor experimentally show a difference. In a dynamic simulation (Figure $7 \mathrm{~b}$ ) however, an angle mismatch of $8.5^{\circ}$ shows a decrease in modulus of up to $50 \mathrm{MPa}$.

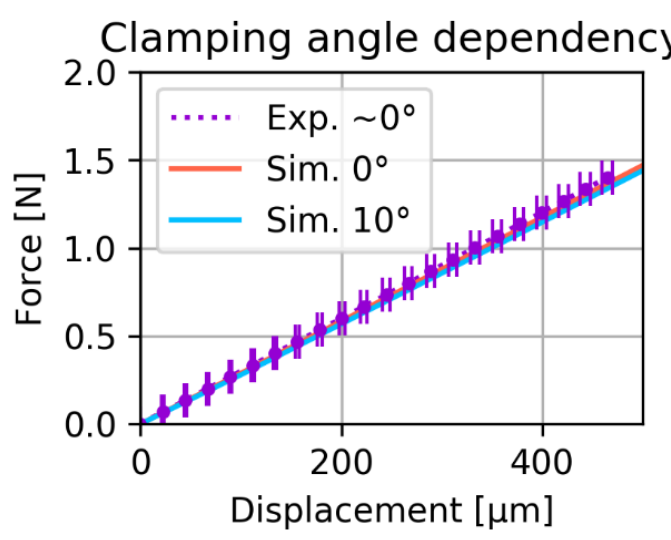

(a)

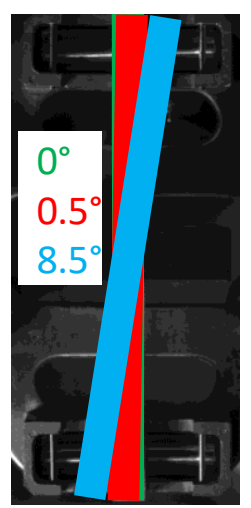

Dynamic simulation

\begin{tabular}{l|l}
\hline Angle & E'$^{\prime}[\mathrm{MPa}]$ \\
\hline $0^{\circ}$ & 2842.7 \\
\hline $0.5^{\circ}$ & 2842.5 \\
\hline $8.5^{\circ}$ & 2794.2
\end{tabular}

(b)

Figure 7: Overview of the influence of the sample misalignment, showing the results of static and dynamic $(1 \mathrm{~Hz}, 500 \mu \mathrm{m})$ simulations and experiments. Results of static testing $(0.2 \mathrm{~N} / \mathrm{min})$ as (a) force- 
displacement-curve of simulations and experiments dependent on rotation angle and (b) dynamic results depending on the visualised angle of the clamped sample, generated with FE

\subsubsection{Repeating measurements}

In DMA, it is possible to program the same loading cycle several times in one experiment. It was observed that the first measured cycle was higher in modulus than the following cycles. In a frequency sweep from 1-25 Hz the same loading cycle was applied 100 times (Figure 8). Of the 100 cycles, the first one deviates significantly more (15.7 MPa) from the next cycle, while the subsequent sequences gradually converge. An unsteady first cycle also occurred in amplitude sweeps. However, between the second and third cycle, the difference becomes small (difference of 2.4 MPa over the whole frequency range), and further cycling is not required to determine an accurate value. In addition, the gradual drop in modulus is typical for material relaxation. However, it was verified that the samples fully recovers after a relaxation and a second test on the same samples shows the same values and behaviour.

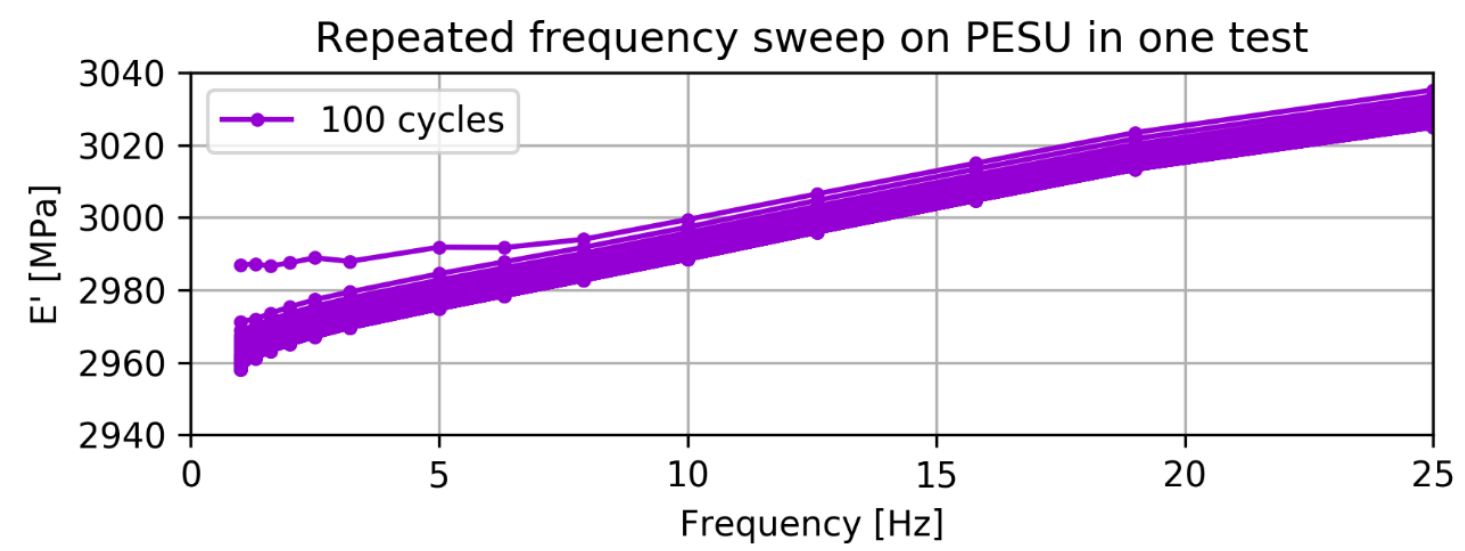

Figure 8: Frequency sweep on PESU, no initiation time like isothermal step, sweep from 1-25 Hz, 100 cycles within the same test.

The reason for the divergent first cycle can be found when observing the displacement with Digital Image Correlation (DIC) at $25 \mathrm{fps}$ (Figure 9). Here, a test at $1 \mathrm{~Hz}$ is repeated seven times within one run. Each cycle consists of four stabilisation loops and three averaging loops from which the moduli are calculated (standard setting for Q800 DMA). The measurements show that between each cycle, the machine reduces the amplitude and restarts for the next cycle. The required amplitude of $500 \mu \mathrm{m}$ is reached at the first cycle, but the static force, that causes a certain displacement to hold the sample in place, is still adjusting during the first measurement cycle, therefore the absolute displacement slightly changes for the following cycles. This is most likely causing the deviating storage modulus between the first and the subsequent cycles 
in cyclic measurement sweeps as in previous Figure 8. Note that the displacement during the four averaging loops varies $20 \mu \mathrm{m}$, which was due to the out-of-plane inaccuracy of the DIC.

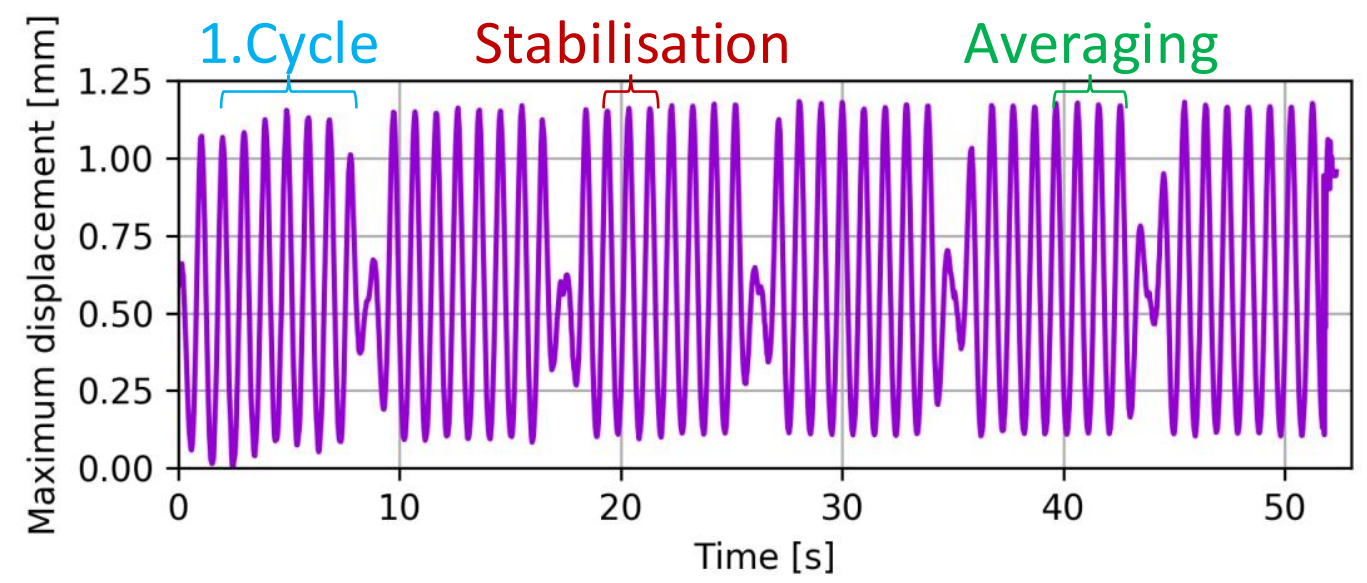

Figure 9: DIC footage of a frequency sweep at $1 \mathrm{~Hz}$. Seven sweeps within one test on a PESU sample, amplitude $500 \mu \mathrm{m}$. Each cycle contains three stabilisation and four averaging loops

\subsubsection{Repetitions on different samples}

As in every other mechanical test, a representative result for a batch of materials should be the average of the results from the same repeated test. In DMA, it depends on the type of sweep how many repetitions are needed for a quantitative result. One test is for example sufficient, if amplitude or frequency sweeps are used only to identify valid test regions as these ranges are much larger than the variation expected within one material. For a quantitative use of amplitude and frequency sweeps, however, the amount of tests is relevant. Therefore, it was tested how many results from different tests need to be combined to receive the same average value. The considered data set contained 20 test results, generated by testing different samples with comparable settings. To know how many repetitions are necessary, combinations of different tests were formed and the standard deviation compared. From the 20 available test results, 2 results were randomly taken (allowing repetitions) and the standard deviation calculated. This was done 50 times (grey curve at "number of considered tests" $=2$ in Figure 10). From those 50 standard deviations, the overall standard deviation was calculated again, forming the first dot of the red curve. The same procedure was repeated with higher numbers of taken values ( 3 to 20 ), forming in total 50 grey random curves and one red standard deviation curve.

Both plots show a greater variation in standard deviation if $\mathrm{x}$ is small. The different curves converge with an increasing number of $\mathrm{x}$. Thereby the standard deviation improves steeply for a smaller $\mathrm{x}$ and flattens out at higher numbers. For amplitude sweeps, it seems to take around 
thirteen tests to have a very stable standard deviation, but already at around five repetitions, the curve is flattening and the improvement with adding further tests is decreasing with the amount of tests added. For the frequency sweep, the curve starts to flatten at six repetitions and stabilises at eight. For cost-efficient testing five amplitude sweeps and six frequency sweeps can be advised. That way the standard deviation stays the same, no matter which different test combination was chosen.

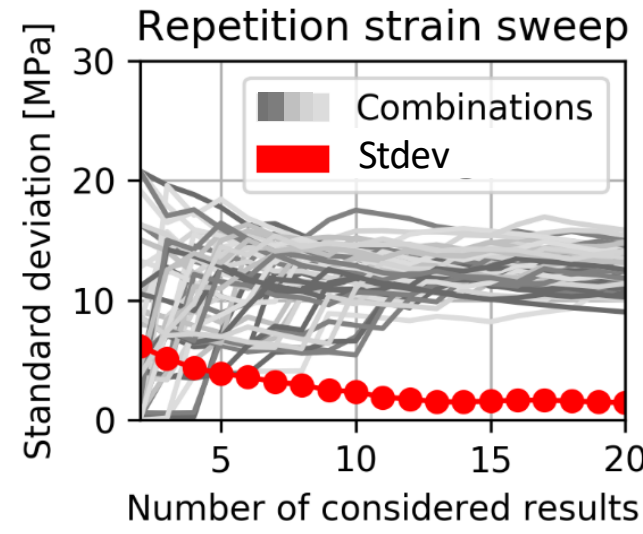

(a)

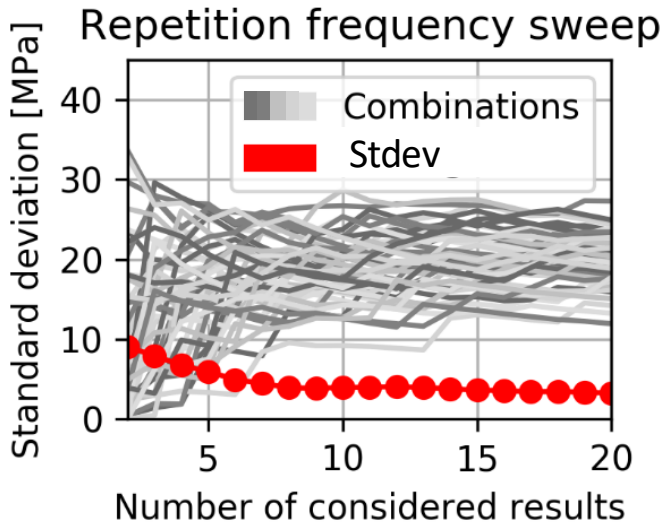

(b)

Figure 10: Randomly combined test data from the same 20 tests: a) amplitude sweep results at $50 \mu \mathrm{m}, \mathrm{b})$ from frequency sweep at $1 \mathrm{~Hz}$ to determine how many tests are statistically required for a repeatable test result. The red line shows the variation between different combinations, the lower, the higher the repeatability

\subsection{Choice of material-specific testing parameters}

\subsubsection{Settings for PESU}

The amplitude sweep should be performed to find the area of viscoelastic response. The viscoelastic region is recognizable from a constant storage modulus over increasing amplitude and a linear increase of stress [2]. Figure 11 shows five repeated strain sweeps with different amplitude ranges. All tests show a linear viscoelastic response of the sample between 0.01$0.3 \%$ strain, respectively an amplitude of $20-650 \mu \mathrm{m}$, resulting in a measured modulus of $2943 \pm 32 \mathrm{MPa}$. At lower amplitudes, the response is in the noise level range of the measurement, and thus the measurement is not accurate. At larger amplitudes, the viscoelastic region is exceeded, which can be noticed by the drop in storage modulus and the stress-strain curve deviating from the linear curve. 


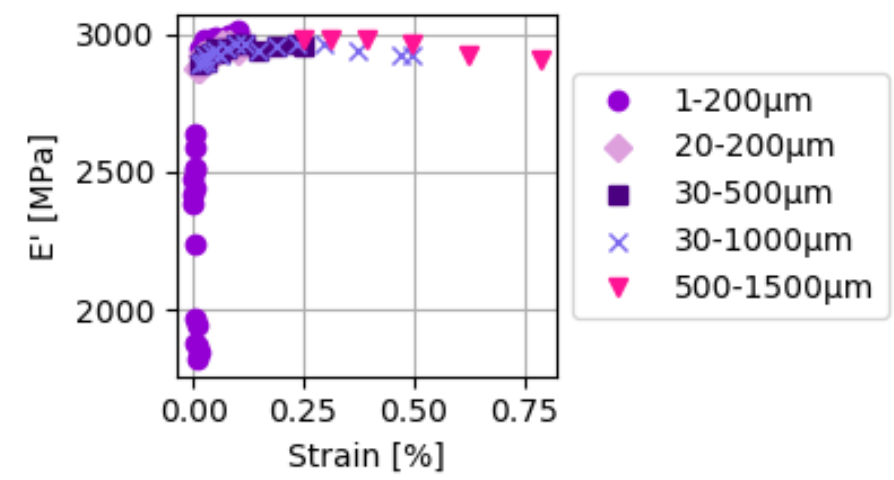

(a)

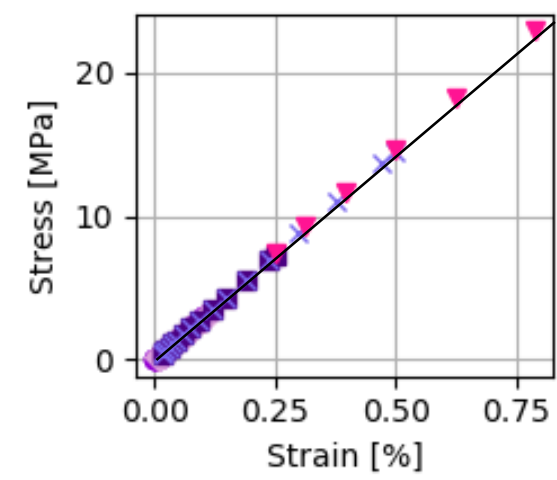

(b)

Figure 11: Amplitude sweep of PESU with aspect ratio 25, five repetitions, but with different amplitudes. (a) storage modulus, (b) stress over strain with black linear trend line.

Further analysis showed that amplitudes at the higher end of the linear viscoelastic range are preferred, since the noise on the result is lower, $1.5 \%$ for $0.25 \%$ strain amplitude in comparison to $4.2 \%$ for $0.01 \%$ strain amplitude (Figure 12). For the PESU specimens this $0.25 \%$ bending strain corresponds to an amplitude of $500 \mu \mathrm{m}$.

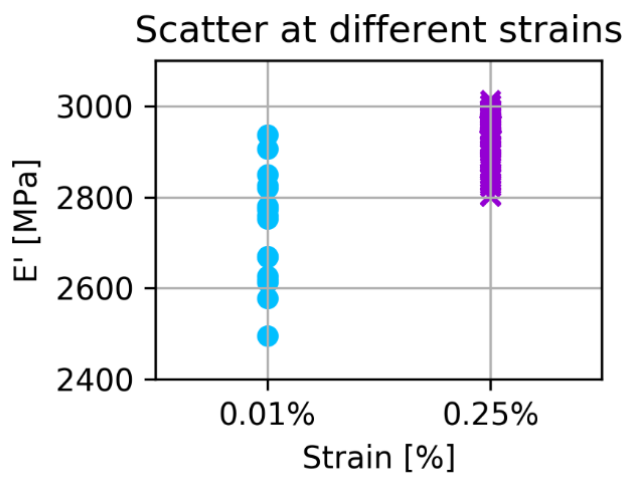

Figure 12: Comparison between small $(0.01 \%, 20 \mu \mathrm{m})$ and big $(0.25 \%, 500 \mu \mathrm{m})$ amplitude within valid test range for $\mathrm{PESU}$ at $1 \mathrm{~Hz}$

The combination of sample, fixture, and frequency can lead to unstable areas of resonance. To detect those frequency values, a frequency sweep can be performed. Figure 13 shows a frequency sweep up to $50 \mathrm{~Hz}$ on PESU material. Indications for resonance are sudden changes in or negative values of the moduli. Two 3-point-bending fixtures, which differ mainly in span length, were compared to illustrate the sensitivity of the system. The $15 \mathrm{~mm}$ fixture shows smooth, valid curves until $40 \mathrm{~Hz}$, the $50 \mathrm{~mm}$ fixture until $20 \mathrm{~Hz}$. For higher frequency values until $50 \mathrm{~Hz}$, the resonance falsifies the result of the modulus. Despite the bigger frequency range, the $15 \mathrm{~mm}$ fixture is not recommended, since the low span to thickness ratio approaches the sensitivity limits of the machine and leads to a lower storage modulus, which was also confirmed by the FE-model. 


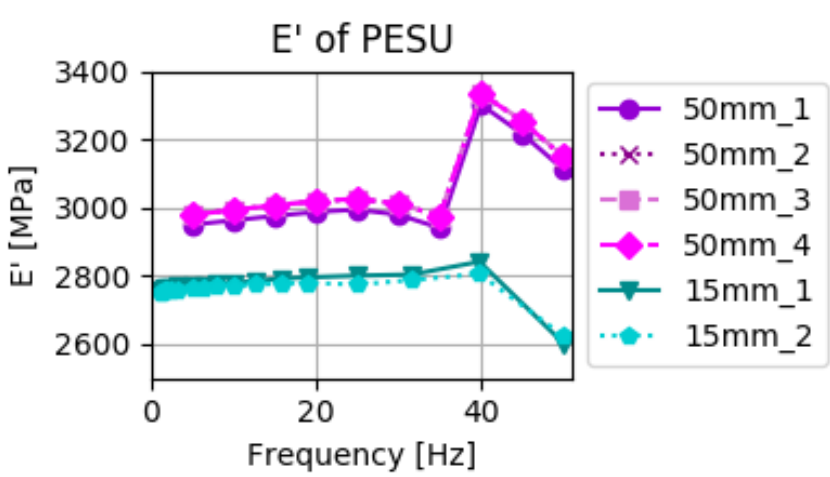

(a)

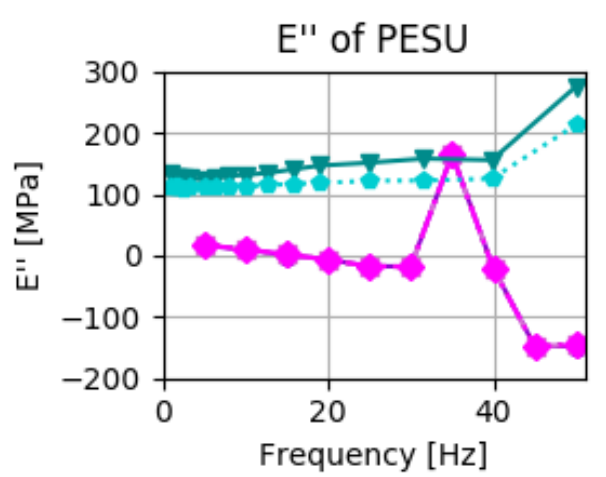

(b)

Figure 13: Inconsistency of storage (a) and loss (b) modulus at higher frequencies due to resonance because of high sample stiffness for 15 and $50 \mathrm{~mm}$ span fixtures at $0.25 \%$ strain, experiment repeated on six different samples

\subsubsection{Settings for Technoform-PP}

The above drawn conclusions were also applied on a much stiffer composite material, being a highly graphite-filled PP (Technoform-PP), with a slightly curved surface due to the specimens being cut from a tubular section. The influence of the width was tested on these samples deviating in shape from a rectangular beam (Figure 14). Samples of $2.9 \mathrm{~mm}$ width were compared with $5.7 \mathrm{~mm}$ wide samples. The average of wide samples is here significantly higher, because of the bigger arc. The narrow Technoform-PP samples have a scatter of $8.3 \%$, while the wide samples have a scatter of $10.0 \%$. Therefore, narrow samples were used for the following sweeps.

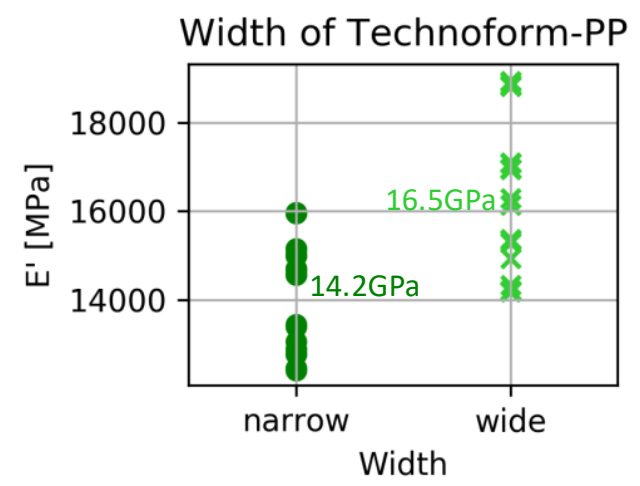

Figure 14: Effect of the sample width on the storage modulus. Each symbol corresponds to a measurement on a single specimen ( 20 per width), average written next to group.

The amplitude sweep should only serve qualitative purpose to find the valid amplitude region at $1 \mathrm{~Hz}$. The test in Figure 15 is showing a linear, stable behaviour only in the small strain region, namely between $0.004 \%$ and $0.011 \%$, respectively $10 \mu \mathrm{m}$ and $25 \mu \mathrm{m}$. 


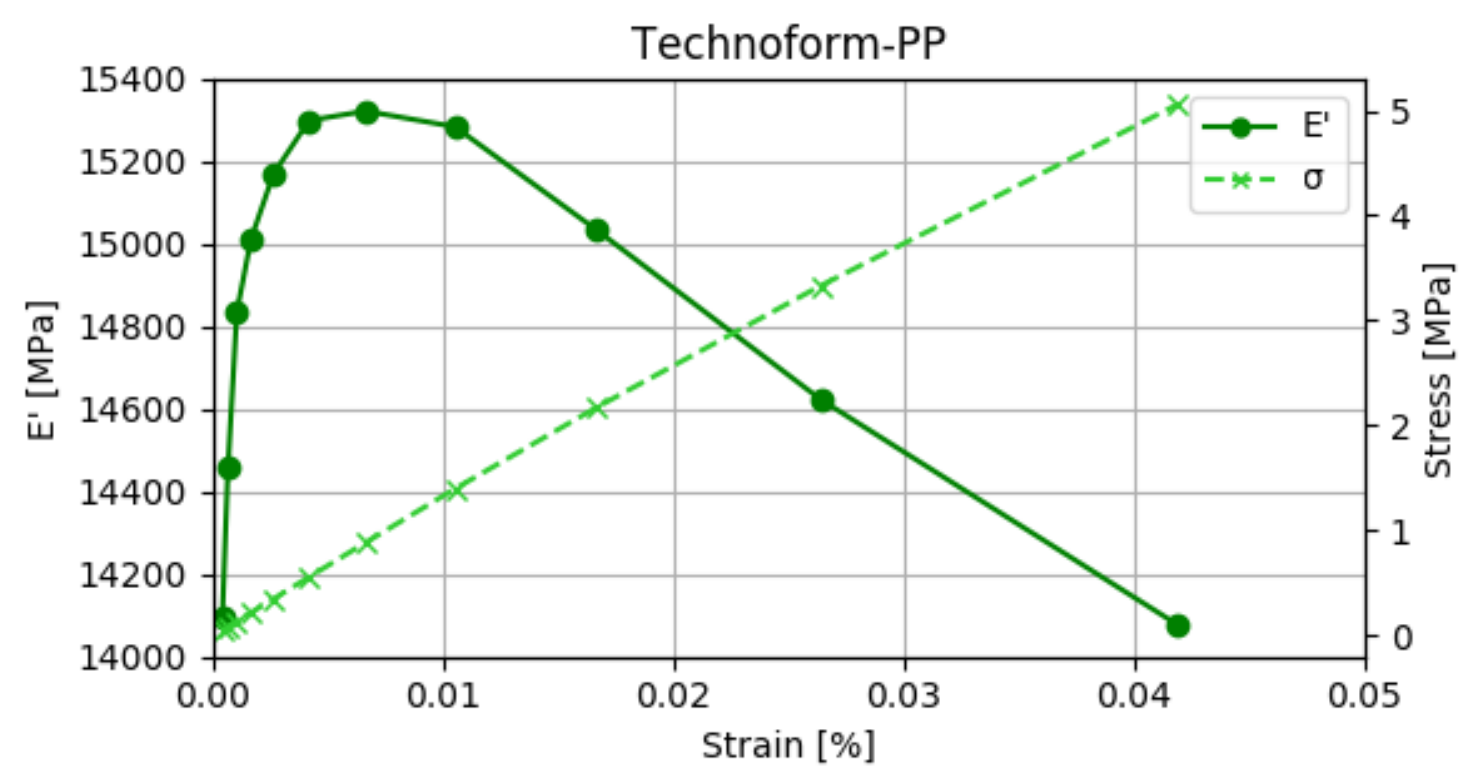

Figure 15: Amplitude sweep of Technoform-PP, St-Ratio 25, $1 \mathrm{~Hz}$; Plotting storage modulus and stress over strain to interpret valid test region

A frequency sweep at $0.01 \%$ strain was performed to evaluate the scatter of results (Figure 16).

The storage modulus shows a smooth course for all tests and the loss modulus stays positive.

Due to the different specimen stiffness and geometry, the complete 1-30 Hz range was stable. The scatter on the storage modulus per frequency is with $1.7 \%$ smaller than the averaged scatter of $2.6 \%$ on the loss modulus. At $1 \mathrm{~Hz}_{2}$ the storage modulus is $15.27 \pm 0.26 \mathrm{GPa}$ and the loss modulus is $0.58 \pm 0.02 \mathrm{GPa}$.

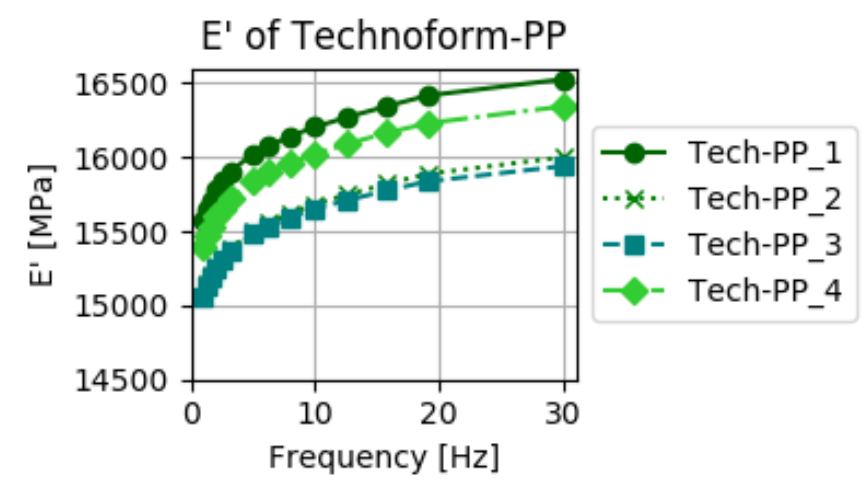

(a)

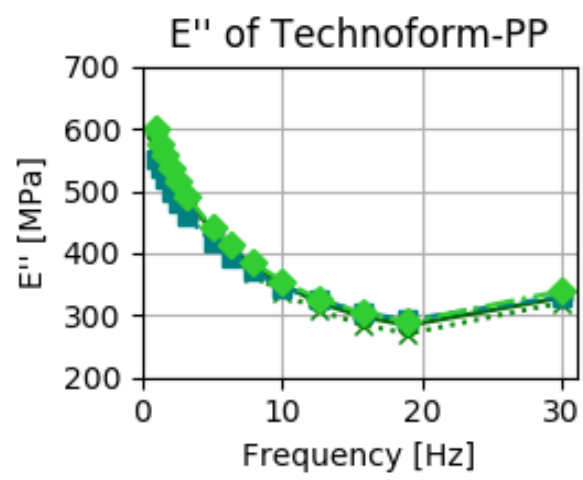

(b)

Figure 16: Storage (a) and loss (b) modulus in frequency sweep of Technoform-PP, $35^{\circ} \mathrm{C}$, experiment repeated on four different samples

A temperature scan on Technoform-PP (Figure 17) shows the temperature dependence of the polypropylene based composite. Within $5{ }^{\circ} \mathrm{C}$ difference, the storage modulus drops $5 \%$ $(0.70 \mathrm{GPa})$. Therefore, a stable temperature is very important during the testing of temperature 
sensitive material. The peak visible on the curve of the loss modulus shows a material transition [24].

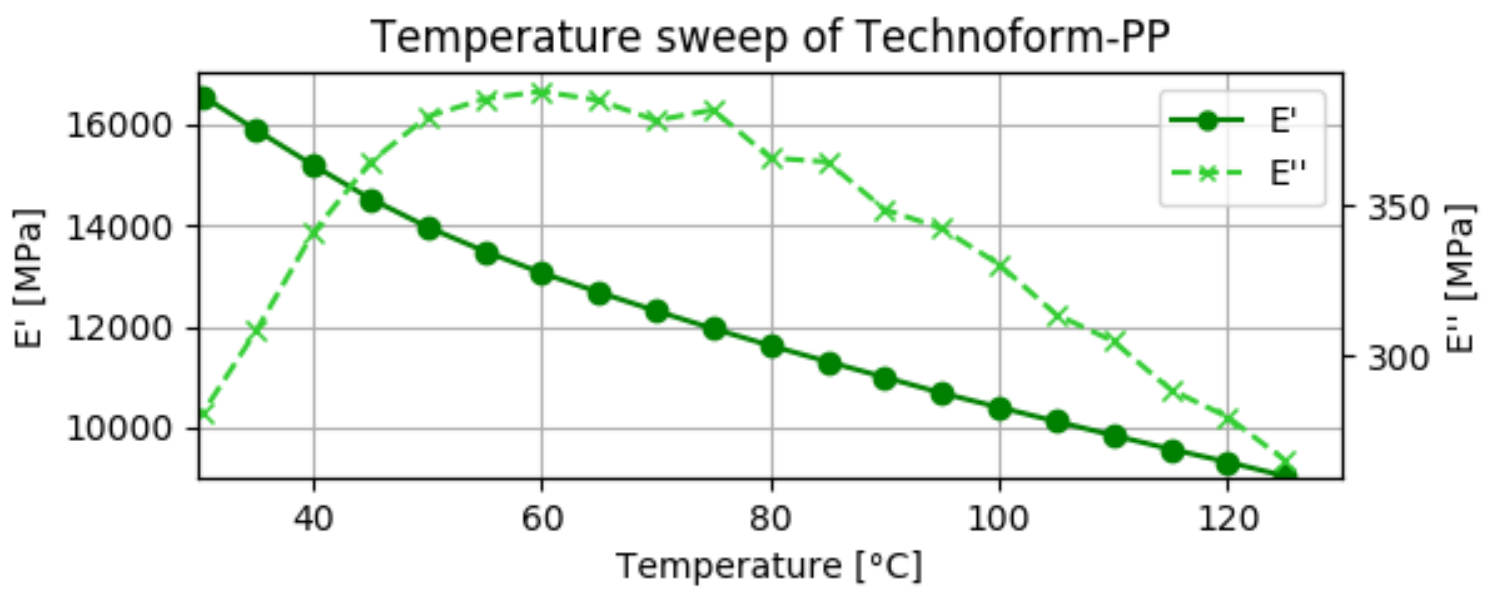

Figure 17: Temperature sweep of Technoform-PP, $1 \mathrm{~Hz}, 20 \mu \mathrm{m}$, modulus over temperature to show temperature dependency of material between $30-130{ }^{\circ} \mathrm{C}$

With this information on the linear viscoelastic region, the frequency range, and the temperature range, it would for example be possible to perform sweeps for time-temperature superposition.

\subsubsection{Influence of recommended settings}

In literature, settings for machine and sample are given in a generic way. This study suggests a procedure to adapt settings depending on the tested material. To compare the effect, tests on PESU were performed in three different settings (Figure 18):

- Arbitrary: span-to-thickness (ST)-ratio below 10; load until upper machine limit; frequency and amplitude from the full range of the machine; fast heating rates $\left(>5^{\circ} \mathrm{C} / \mathrm{min}\right)$

- Machine-specific: ST-ratio of 25; stress, strain, and frequency ranges within the machine-specific recommended boundaries; low heating rate $\left(2^{\circ} \mathrm{C} / \mathrm{min}\right)$

- Material-specific: valid ST-ratio of 25; stress, strain, and frequency ranges according to material requirements; low heating rate $\left(2^{\circ} \mathrm{C} / \mathrm{min}\right)$

The results of this study, adapted to machine and material, show a scatter of only $0.6 \%$ and have no significant outliers. Tests following settings recommended in literature have a broader distribution of measured values, resulting in a scatter of $3.7 \%$ on the average storage modulus, but deliver results in the correct order of magnitude, slightly underestimating the stiffness. The results of arbitrary tests however have a scatter of $17.6 \%$ and cause outliers in both directions with a difference of up to $60 \%$ compared to the average modulus. Hence, they do not deliver 
trustworthy results without an extensive study to eliminate the outliers. This shows that in order to use DMA test data to quantify the viscoelastic nature or long-term material response of materials, very specific guidelines should be followed. Moreover, this study was performed on a high-grade temperature and moisture stable material, and other polymers might be even more sensitive.

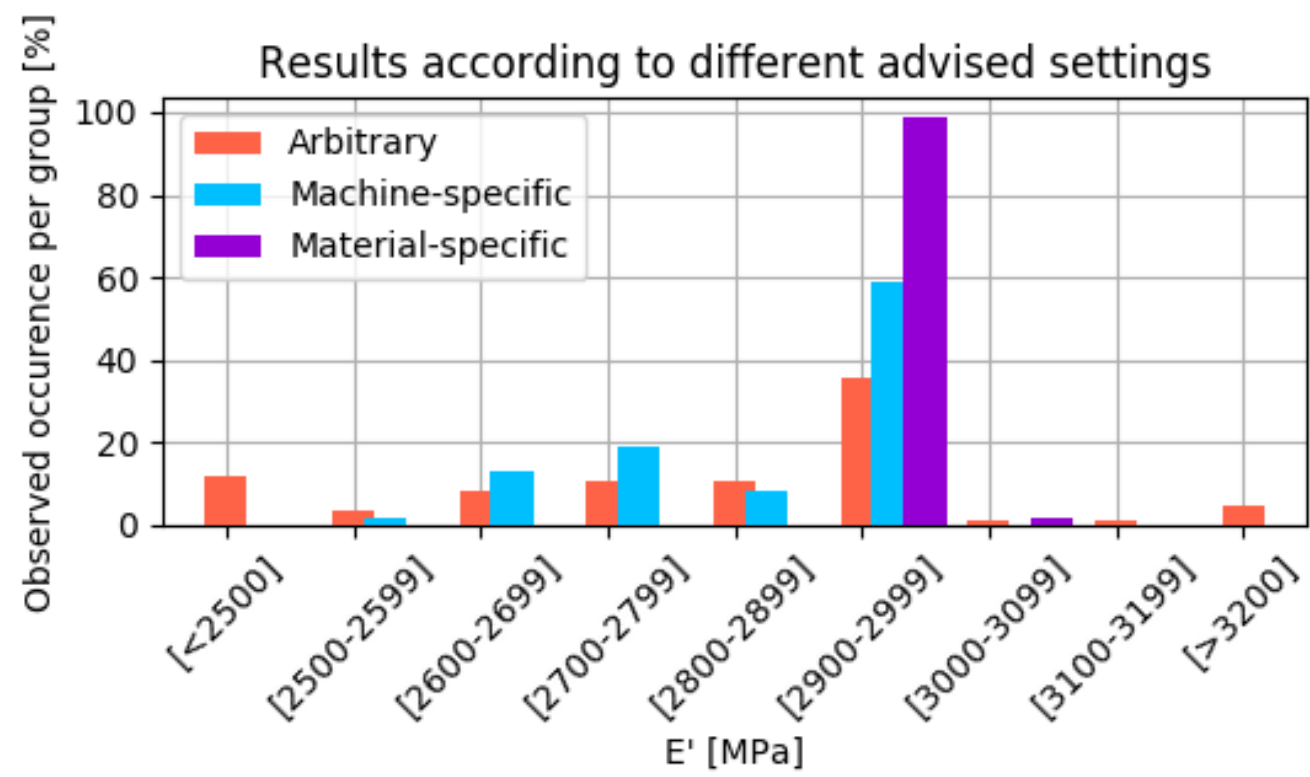

Figure 18: Distribution of results depending on the settings. Experiments using PESU, $1 \mathrm{~Hz}$ in different bending fixtures. Tests according to recommendations of this study (material specific) were compared to tests according to machine-specific settings described in literature and to tests aribrary chosen from the possible settings. Each colour-group contains more than 60 samples

\section{Discussion and conclusions}

Dynamic mechanical analysis (DMA) in a 3-point-bending setup allows studying the linearviscoelastic region of materials. In this study, PESU has been tested to determine, which factors and parameters easily increase the scatter of repeated measurements and therewith make the results less useable in quantitative sense. The developed measurement system has successfully been applied to the graphite filled composite Technoform-PP. The testing scatter for Technoform-PP samples resulted with the settings of this study to $1.7 \%$. For PESU samples, the dimensional accuracy was with $1.6 \%$ the limiting factor even though an accuracy of $0.6 \%$ was achieved in the tests for this study. The experimental scatter could be kept low, if following guidelines are respected:

- The accuracy in dimensions should at least be as precise as $\pm 2 \%$.

- The span-to-thickness ratio should be higher than 10 . 
- The width is not limited as long the contact pressure can be ensured over the full width and precision can be assured. In case of not perfectly flat samples, smaller widths are advised.

- The alignment mismatch between the specimen's centreline and the fixture can be neglected for mismatch angles coming from specimen aligning by the naked eye.

- The limitations in frequency and amplitude cannot be given generically, but require material and setup specific adjustments. For new materials or material in new dimensions, a testing procedure was recommended above, and is summarised here:

- The first cycle of a sweep does not appear as stable as results from the second cycle on. It is recommended to have at least one iteration within one test and take the results of the last iteration only.

- An amplitude sweep determines the linear viscoelastic region. From the range of possible amplitudes, the highest should be chosen for stable results. For determination of the viscoelastic region in a homogeneous material, a single sweep is sufficient, to use the modulus quantitatively, at least five but ideally twelve repetitions are recommended.

- A single frequency sweep can be used to detect resonances, however, to use the modulus quantitatively, at least six and ideally thirteen repetitions are recommended.

The results of this study should allow for sufficient boundary conditions for the test settings to enable repeatable, accurate measurement of the storage and loss modulus that can serve for long-term predictions and calibration of numerical models.

\section{Acknowledgements}

The authors acknowledge the financial support from EIT Raw Materials project "RELICARIO" under Grant Agreement No. 18239. This research also received funding from Agentschap Innoveren \& Ondernemen (Vlaio/FWO) SBO project "COMPOHEX" under grant agreement 150013.

\section{Data availability}

The raw/processed data required to reproduce these findings cannot be shared at this time as the data also forms part of an ongoing study. The data is available on request. 


\section{References}

[1] Mettler-Toledo GmbH, Analytical, "Thermal Analysis in Practice: Tips and Hints", 2016.

[2] TA Instruments, "Dynamic mechanical analysis", 2016.

[3] J. Dealy and D. Plazek, "Time-temperature superposition - a users guide", Rheology Bulletin, vol. 78, no. 2, pp. 16-31, 2009.

[4] P. Lee-Sullivan and D. Dykeman, "Guidelines for performing storage modulus measurements using the TA Instruments DMA 2980 three-point bend mode - I. Amplitude effects", Polymer testing, vol. 19, pp. 199-164, 2000.

[5] C. Grein, K. Bernreitner, and M. Gahleitner, "Potential and Limits of Dynamic Mechanical Analysis as a Tool for Fracture Resistance Evaluation of Isotactic Polypropylenes and Their Polyolefin Blends", Wiley InterScience, 2004.

[6] ASTM D4065: "Plastics: Dynamic Mechanical Properties: Determination and Report of Procedures", ASTM International, 2012.

[7] A. Margossian, S. Bel, and R. Hinterhoelzl, "Bending characterisation of a molten unidirectional carbon fibre reinforced thermoplastic composite using a Dynamic Mechanical Analysis system", Composites: Part A, vol. 77, pp. 154-163, 2015.

[8] G. Swaminathan, K. N. Shivakumar, and L. C. Russell Jr, "Anomalies, influencing factors, and guidelines for DMA testing of fiber reinforced composites", Polymer Composites, vol. 30, no. 7, pp. 962-969, 2009.

[9] M. Tajvidi, R. H. Falk, and J. C. Hermanson, "Time-temperature superposition principle applied to a kenaf-fiber/high-density polyethylene composite", Journal of Applied Polymer Science, vol. 97, no. 5, pp. 1995-2004, 2005.

[10] T. H. Lee, F. Y. C. Boey, and N. L. Loh, "Characterization of a fibre-reinforced PPS composite by dynamic mechanical analysis: Effect of aspect ratio and static stress", Composites Science and Technology, vol. 49, pp. 217-223, 1993.

[11] B. Brylka, M. Schemmann, J. Wood, and T. Böhlke, "DMA based characterization of stiffness reduction in long fiber reinforced polypropylene", Polymer Testing, vol. 66, pp. 296-302, 2018.

[12] TA Instruments, "DMA Online Help", Waters LLC, 109 Lukens Drive, New Castle, DE 19720.

[13] ASTM D790-10, "Standard Test Methods for Flexural Properties of Unreinforced and Reinforced Plastics and Electrical Insulating Materials", ASTM international, 2010.

[14] ASTM D5023-15, "Standard Test Method for Plastics: Dynamic Mechanical Properties: In Flexure (Three-Point Bending)", ASTM International, 2015.

[15] ASTM D7028-15, "Glass Transition Temperature (DMA Tg) of Polymer Matrix Composites by Dynamic Mechanical Analysis (DMA)", ASTM International, 2015.

[16] DIN EN ISO 6721-1, "Plastics - Determination of dynamic mechanical properties. Part 1: General principles", ISO, 2011.

[17] I. M. McAninch, G. R. Palmese, J. L. Lenhart, and J. J. La Scala, "DMA testing of epoxy resins: The importance of dimensions", Polymer Engineering and science, vol. 55, no. 12, pp. 2761-2774, 2015.

[18] Mettler-Toledo AG, "Dynamic Mechanical Analysis - Sets New Standards", MettlerToledo AG, Analytical CH-8603 Schwerzenbach, Switzerland, 2014.

[19] PerkinElmer, "A Beginner's Guide: Dynamic Mechanical Analysis", 2008.

[20] ASTM D5026, "Standard Test Method for Plastics: Dynamic Mechanical Properties: In Tension", ASTM International, 2015.

[21] PerkinElmer, "DMA 800", 2013.

[22] TA Instruments, "Thermal analysis - Q800 Specifications", 2010. 
[23] Q. Shao and P. Lee-Sullivan, "Guidelines for performing storage modulus measurements using the TA Instruments DMA 2980 three-point bend mode - II. Contact stresses and machine compliance", Polymer Testing, vol. 19, pp. 239-250, 2000.

[24] P. Zhang and T. Kraus, "Anisotropic nanoparticles as templates for the crystalline structure of an injection-molded isotactic polypropylene/TiO2 nanocomposite", Polymer, vol. 130, pp. 161-169, 2017.

[25] Solvay, "Veradel A-301 polyethersulfone", Solvay Specialty Polymers, 2014.

[26] Solvay, "Radel@ PPSU, Veradel® PESU \& Acudel® modified PPSU: Design Guide, Version 6", 2014.

[27] J. Caprano, "Technoform Heat transfer solutions," Technoform Kunststoffprofile GmbH, 2019.

[28] TA Instruments, "2980 DMA Stress-Strain Equations", 2002.

[29] Correlated Solutions, "Vic-3D manual", Columbia, SC, 2010.

[30] Dassault Systems Simulia Corp, "Abaqus User Documentation", RI, USA, 2017. 